\title{
Slip Reduction Rate between Minimal Invasive and Conventional Unilateral Transforaminal Interbody Fusion in Patients with Low-Grade Isthmic Spondylolisthesis
}

\author{
Chang Hyun Oh${ }^{1}$, Gyu Yeul $\mathrm{Ji}^{2}$, Jae Kyun Jeon", Junho Lee ${ }^{1}$, \\ Seung Hwan Yoon ${ }^{3}$, Dong Keun Hyun ${ }^{3}$ \\ ${ }^{I}$ Department of Neurosurgery, Guro Teun Teun Hospital, Seoul, \\ ${ }^{2}$ Department of Neurosurgery, Yonsei University College of Medicine, Seoul, \\ ${ }^{3}$ Department of Neurosurgery, Inha University College of Medicine, Incheon, Korea
}

\begin{abstract}
Objective: To compare the slip reduction rate and clinical outcomes between unilateral conventional transforaminal lumbar interbody fusion (conventional TLIF) and unilateral minimal invasive TLIF (minimal TLIF) with pedicle screw fixation for treatment of one level low-grade symptomatic isthmic spondylolisthesis.

Methods: Between February 2008 and April 2012, 25 patients with low-grade isthmic spondylolisthesis underwent conventional TLIF (12 patients) and minimal TLIF (13 patients) in single university hospital by a single surgeon. Lateral radiographs of lumbar spine were taken 12 months after surgery to analyze the degree of slip reduction and the clinical outcome. All measurements were performed by a single observer.

Results: The demographic data between conventional TLIF and minimal TLIF were not different. Slip percentage was reduced from $15.00 \%$ to $8.33 \%$ in conventional TLIF, and from $14.15 \%$ to $9.62 \%$ in minimal TLIF. In both groups, slip percentage was significantly improved postoperatively $(\mathrm{p}=0.002)$, but no significant intergroup differences of slip percentage in preoperative and postoperative were found. The reduction rate also not different between conventional TLIF $(45.41 \pm 28.80 \%)$ and minimal TLIF (32.91 $\pm 32.12 \%, \mathrm{p}=0.318)$.

Conclusion: Conventional TLIF and minimal TLIF with pedicle screw fixation showed good slip reduction in patients with one level low-grade symptomatic isthmic spondylolisthesis. The slip percentage and reduction rate were similar in the conventional TLIF and minimal TLIF.
\end{abstract}

Key Words: Slip percentage $\cdot$ Reduction rate $\cdot$ Conventional $\cdot$ Minimal invasive $\cdot$ Transforamenal lumbar interbody fusion

\section{INTRODUCTION}

Spondylolisthesis is an unstable lumbar disease demonstrated in approximately $6 \%$ of the general population and occurs most often at L4-L5 and L5-S1 levels ${ }^{10,13,14)}$. Anatomy research indicates that isthmic spondylolisthesis usually occurs within

- Received: September 5, 2013 • Revised: November 18, 2013

- Accepted: November 21, 2013

Corresponding Author: Seung Hwan Yoon, MD, PhD

Department of Neurosurgery, Inha University Hospital, College of Medicine, Inha University, 7-206, 3-Ga, Shinheung-dong, Jung-gu, Incheon 400-711, Korea

Tel: +82-32-890-2370, Fax: +82-32-890-2374

E-mail: nsyoon@gmail.com

@This is an Open Access article distributed under the terms of the Creative Commons Attribution Non-Commercial License (http://creativecommons.org/ licenses/by-nc/3.0/) which permits unrestricted non-commercial use, distribution, and reproduction in any medium, provided the original work is properly cited. the scope of 2 to $9 \mathrm{~mm}$ beneath the pedicles, resulting in the defects in bony contacts in the pars interarticularis to form pseudarthrosis, the gap of which is filled with fibrous connective tissue and chondroid tissue, which we called pars interarticularis scar ${ }^{3)}$. Symptomatic patients with isthmic spondylolisthesis usually require surgical intervention, the goals of which include the stabilization of the motion segment, the decompression of neural elements, the reconstitution of disc space height, and the restoration of sagittal plane translational and rotational alignment ${ }^{6}$. Minimally invasive transforaminal lumbar interbody fusion (TLIF) is a relatively novel spinal fusion technique which was developed in recent years on the basis of conventional TLIF ${ }^{3)}$. But, little is known about the slip reduction according to the unilateral TLIF methods. This study was to compare the slip reduction rate and clinical outcome between unilateral conventional TLIF (conventional TLIF) with pedicle screw fixation and unilateral minimal invasive TLIF (minimal TLIF) with pedicle screw fixation for treat- 
ment of one level low-grade symptomatic isthmic spondylolisthesis.

\section{MATERIALS AND METHODS}

\section{Clinical material}

Transforaminal lumbar interbody fusion is a relatively new technique of lumbar arthrodesis via posterior transforaminal approach to the disc, indicated mainly in cases of degenerative disc disease, low grade spondylolisthesis and reoperation for disc herniation, especially when there is indication for interbody fusion and posterior decompression. The main advantage of TLIF is that it allows the complete removal of the intervertebral disc through the vertebral foramen, decompression of the spinal canal and vertebral foramen with minimum risk of neural lesion, due to the access being lateral to the nerve roots. Between February 2008 and April 2012, 25 patients with lowgrade isthmic spondylolisthesis underwent conventional TLIF (12 patients) and minimal TLIF (13 patients) in single university hospital by a single surgeon were included in the study-including 6 males and 19 females, whose average age was 51.96 years (21-72 years). All of them had preoperative radiographs, computed tomography scans, as well as magnetic resonance imaging, and all surgical procedures were performed by a single surgeon $(\mathrm{SHY})$.

\section{Assessment of slip reduction and clinical results}

Lateral radiographs of lumbar spine were taken 12 months after surgery to analyze the degree of reduction. All measurements were performed by a single observer and were expressed as means \pm standard deviations. To control for small variations, the radiographic results were represented in percentage. Slip percentage was measured as a percentage of distance from the posterior border of the caudal to the posterior border of the rostral vertebra, normalized to the superior endplate diameter of the former as it presented in Fig. $1^{15}$. Clinical outcome was measured by visual analog scale (VAS; score range: 0 to 10 , with 0 reflecting no pain).

\section{Surgical technique of minimal TLIF}

Fluoroscopy was used to determine the operative level in minimal TLIF technique. The minimal TLIF procedure was performed on the side of radicular symptoms. If both the legs were symptomatic, the approach was from the side of more severe pathology and contralateral lamina and foramina decompressed by a unilateral exposure. An incision was made
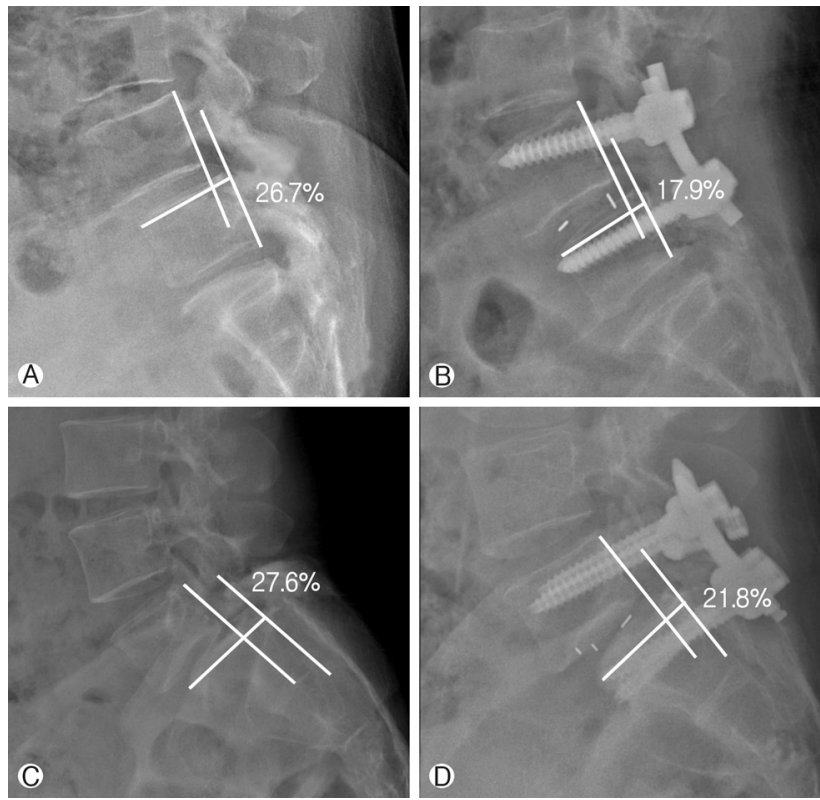

Fig. 1. Slip percentage measured by a percentage of distance from the posterior border of the caudal to the posterior border of the rostral vertebra: slip percentage was decreased from $26.7 \%$ to $17.9 \%$ in conventional TLIF (A and B) and was decreased from $27.6 \%$ to $21.8 \%$ in minimal TLIF (C and D)

3 to $4 \mathrm{~cm}$ off midline. Sequential soft tissue dilators were inserted through the incision down to the facet complex until the desired working diameter was achieved. A facetectomy was then performed using a high-speed drill from lateral to medial side to expose the posterolateral aspect of the disc. Intradiscal distraction and disc space preparation were done using standard interbody fusion instruments. Cartilaginous material was removed from the endplates using the endplate scraper. An interbody graft was then placed in a direction anterior and contralateral to the annulotomy within the interbody space. Autograft was not used in any cases. Fluoroscopy was used to ensure satisfactory placement of the graft. When necessary, the contralateral ligamentumflavum was resected to expose the contralateral exiting and traversing nerve roots. If needed, the tubular retractor was angled contralaterally so that a more extensive boney decompression could be done. The tubular retractor was then removed and percutaneous pedical screws placed immediately above and below the interbody segment to be fused. Under fluoroscopic guidance, a Jamshidi needle was inserted into the pedicles. A K-wire was then passed through the Jamshidi trocar into the pedicles. Using cannulated instruments, a bone tap followed by cannulated screw was advanced over the K-wire. The rod was then placed percutaneously to connect the screws. Compression was applied to the construct before final tightening, providing compression of the bone graft and maximizing lordosis. All 
wounds were copiously irrigated and the wounds were closed in layers.

\section{Surgical technique of conventional TLIF}

A midline skin incision was used in conventional TLIF. The fascia was incised and the paravertebral muscles were dissected from the spine. Radiographs were used to check the appropriate level. Bilateral pedicle screw rod constructs were inserted and laminectomy and unilateral facetectomy was then performed at that level. This was followed by unilateral anulotomy, discectomy, and placement of the interbody graft. Similar to the minimal TLIF approach, cartilaginous material was removed from the endplates using the endplate scraper. Interbody graft was then placed anteriorly and contralateral to the annulotomy within the interbody space. For posteriorlateral arthodesis, local autogenous bone with or without bone extenders was used for bone grafting. The wound was copiously irrigated and closed in layers.

\section{Statistical analysis}

SPSS software for Windows (SPSS Inc., Chicago, IL, USA) was used throughout, and statistical significance was accepted for $\mathrm{p}$ values of $<0.050$. Data were analyzed using the student, student paired t-test, and chi-square test.

\section{RESULTS}

The demographic data between conventional and minimal TLIF were not different (Table 1). All the cases were followed up more than 12 months. Slip percentage was reduced from $15.00 \pm 6.66 \%$ to $8.33 \pm 5.31 \%$ in conventional TLIF, and from $14.15 \pm 3.76 \%$ to $9.62 \pm 5.42 \%$ in minimal TLIF (Table 2). In both groups, slip percentage was significantly improved postoperatively $(\mathrm{p}=0.002)$, but no significant intergroup diffe-

Table 1. Demographic date between convention and minimal TLIF with pedicle screw fixation

\begin{tabular}{lllc}
\hline \hline & Conventional TLIF & Minimal TLIF & p-value \\
\hline No. of Cases & 12 & 13 & - \\
Male Ratio & $25.00 \%$ & $15.38 \%$ & 0.567 \\
Age & $50.67 \pm 11.53$ & $53.15 \pm 11.40$ & 0.593 \\
Level & & & \\
L4/5 & 9 & 8 & 0.891 \\
L5/S1 & 4 & 4 & \\
\hline
\end{tabular}

TLIF, transforaminal lumbar interbody fusion; conventional TLIF, unilateral conventional TLIF with pedicle screw fixation; minimal TLIF, unilateral minimal invasive TLIF with pedicle screw fixation. rences of slip percentage in preoperative and postoperative were found (Table 3 ). The reduction rate also not different between conventional $(45.41 \pm 28.80 \%)$ and minimal TLIF (32.91 $\pm 32.12 \%, \mathrm{p}=0.318)$. No neurologic complications were encountered. There were no signs of instrumentation failure and no visible subsidence of the cages until 12 months follow-up periods. All patients had their symptoms significantly improved, and the VAS decreased from $7.00 \pm 2.69$ before operation to $3.11 \pm 1.86$ at 12 months after operation in conventional TLIF, and from $7.14 \pm 3.15$ before operation to $3.52 \pm 2.24$ at 12 months after operation in minimal TLIF (Table 3). The clinical outcome was not correlated with reduction rate $(\mathrm{p}=0.341)$.

\section{DISCUSSION}

The advent of minimally invasive surgery had provided surgeons new techniques for treating clinical disease ${ }^{7}$. Minimally invasive spine surgery aims to reduce approach related morbidity, while producing clinical outcomes comparable to its open predecessors ${ }^{7}$. One important example of this is the development of minimally invasive techniques for lumbar interbody fusion, including TLIF ${ }^{2,7}$. The minimal invasive TLIF technique, has displayed comparable outcomes to conventional TLIF, while adding the benefits of less approach related mor-

Table 2. Comparison result of preoperative and postoperative slip grade

\begin{tabular}{lccc}
\hline \hline & Preoperative & Postoperative & \multirow{2}{*}{ p-value } \\
& Slip Grade (\%) & Slip Grade (\%) & \\
\hline Conventional TLIF & $15.00 \pm 6.66 \%$ & $8.33 \pm 5.31 \%$ & 0.002 \\
Minimal TLIF & $14.15 \pm 3.76 \%$ & $9.62 \pm 5.42 \%$ & 0.002 \\
\hline
\end{tabular}

conventional TLIF, unilateral conventional transforaminal lumbar interbody fusion with pedicle screw fixation; minimal TLIF, unilateral minimal invasive transforaminal lumbar interbody fusion with pedicle screw fixation

Table 3. Preoperative and postoperative clinical and radiologic result

\begin{tabular}{cccc}
\hline \hline & $\begin{array}{c}\text { Conventional } \\
\text { TLIF }\end{array}$ & Minimal TLIF & $\mathrm{p}$-value \\
\hline Preoperative VAS & $7.00 \pm 2.69$ & $7.14 \pm 3.15$ & 0.519 \\
$\begin{array}{c}\text { Preoperative Slip } \\
\text { Grade (\%) }\end{array}$ & $15.00 \pm 6.66 \%$ & $14.15 \pm 3.76 \%$ & 0.696 \\
Postoperative VAS & $3.11 \pm 1.86$ & $3.52 \pm 2.24$ & 0.265 \\
$\begin{array}{c}\text { Postoperative Slip } \\
\text { Grade (\%) }\end{array}$ & $8.33 \pm 5.31 \%$ & $9.62 \pm 5.42 \%$ & 0.557 \\
Reduction Rate (\%) & $45.41 \pm 28.80 \%$ & $32.91 \pm 32.12 \%$ & 0.318 \\
\hline
\end{tabular}

conventional TLIF, unilateral conventional transforaminal lumbar interbody fusion with pedicle screw fixation; minimal TLIF, unilateral minimal invasive transforaminal lumbar interbody fusion with pedicle screw fixation; VAS, visual analog scale. 
bidity, decreased intraoperative blood loss, and shorter hospital stays ${ }^{9,11)}$. However, critics of the technique have noted that the minimal TLIF has longer operative times and exposes patients to increased fluoroscopic radiation. Over the past decade minimal TLIF has been shown to have a number of benefits, especially with regard to perioperative outcomes. However, it may have its own unique challenges and potential morbidity. Ultimately, comparing the known literature of a conventional TLIF approach to published reports on minimal TLIF will identify the unique risks and benefits associated with each.

Generally, clinical result between conventional and minimal TLIF were reported the similar or superior result in minimal invasive technique ${ }^{4,5,17}$. All preoperative parameters except the operation time were superior in minimal TLIF than the conventional TLIF ${ }^{4,5,17,18,20)}$. Most article were concluded to similar the overall complication rate in two methods ${ }^{2,4,5,7,12,20)}$. But, all authors reported that radiation hazard is less effective in minimal TLIF, and concluded the main disadvantage of minimal $\operatorname{TLIF}^{4,5,17)}$. In radiological study, although many reported using the different criteria about the bone fusion, but all reported studies concluded the fusion rate between two TLIF technique is similar ${ }^{17,18)}$. But, the comparisonal data slip reduction in patients with low-grade spondylolisthesis is lack of data, yet. In this study, the authors compared the slip reduction rate between two different unilateral TLIF with pedicle screw fixation for treatment of one level low-grade symptomatic isthmic spondylolisthesis. To best knowledge to authors, this comparison is not published in literature yet, although the size of data was not sufficient to show the conclusive statistical evidence.

In this small case series about 25 patients with low-grade isthmic spondylolisthesis underwent conventional TLIF or minimal TLIF showed, the slip percentage was significantly improved postoperatively in both TLIF techniques $(p=0.002)$. All patients had their symptoms significantly improved after operation in both groups. But, the slip percentage in pre/postoperative periods and reduction rate after operation also not different between conventional and minimal TLIF. The similar results were also observed in the literatures, but most of them are focused only conventional or minimal TLIF. Recniket al. ${ }^{8)}$ reported that significantly decreased translational slip from $20.7 \%$ to $14.9 \%$ after conventional TLIF in 32 patients with single-level isthmic spondylolisthesis $(\mathrm{p}<0.001)$. Yan et al. $\left.{ }^{16}\right)$ also reported the slip was reduced from $31.4 \pm 8.3$ to $8.2 \pm$ $2.6 \%$, and the average of reduction rate was $75.4 \pm 6.2$ in conventional TLIF. In minimal invasive techniques, Pan et al. ${ }^{3)}$ reported decreased translational slip from $24.2 \%$ to $10.5 \%$ after operation, and Tsahtsarlis et al. ${ }^{19)}$ also reported the anatomical reduction of the spondylolisthesis was complete in 16 patients (69.6\%) and incomplete in seven (30.3\%). This result was similar to this data, but direct comparisonal result is observed in only this presented study. Some authors reported surgical technique to increase the reduction rate among spondylolisthesis, the key technique is circumferential disc or ligament reconstruction which prevent the reduction ${ }^{1)}$. A bilateral traversing and exiting nerve roots decompression is a safer option prior to performing the deformity reduction and fixation, and the proposed minimally invasive technique may help in reducing surgical morbidity and improving postoperative recovery.

The conventional and minimal TLIF showed excellent slip reduction in patients with one level low-grade symptomatic isthmic spondylolisthesis and slip percentage and reduction rate were similar in the conventional and minimal TLIF as we presented. But, as we commented, this study is small case series about 25 patients, so the statistical analysis had an opportunity into the trap of the type I error. Indeed, we could not access the other meaningful clinical outcomes such as Oswestrydisability index (ODI), neurogenic symptom score (NSS) andshort form 36 (SF-36) because of using retrograde data review. In the statistical analysis with tripling of this data showed statistical difference of reduction rate $(\mathrm{p}=0.018)$, so well-designed longitudinal prospective study with a larger number of isthmic spondylolisthesis could presented the different reductional result between conventional and minimal TLIF.

\section{CONCLUSION}

The conventional and minimal TLIF with pedicle screw fixation showed excellent slip reduction in patients with one level low-grade symptomatic isthmic spondylolisthesis. The slip percentage and reduction rate were similar in the conventional and minimal TLIF.

\section{REFERENCES}

1. Barbagallo GM, Certo F, Sciacca G, Albanese V: Bilateral tubular minimally invasive approach for decompression, reduction and fixation in lumbosacral lythicspondylolisthesis. Neurosurg Focus 35: Video 9, 2013

2. Dhall SS, Wang MY, Mummaneni PV: Clinical and radiographic comparison of mini-open transforaminal lumbar interbody fusion with open transforaminal lumbar interbody fusion in 42 patients with long-term follow-up. J Neurosurg Spine 9:560-565, 2008

3. Foley KT, Holly LT, Schwender JD: Minimally invasive lumbar fusion. Spine (Phila Pa 1976) 28:S26-35, 2003

4. Habib A, Smith ZA, Lawton CD, Fessler RG: Minimally invasive transforaminal lumbar interbody fusion: a perspective on current evidence and clinical knowledge. Minim Invasive Surg 2012: 657-343, 2012

5. Kalichman L, Kim DH, Li L, Guermazi A, Berkin V, Hunter 
DJ: Spondylolysis and Spondylolisthesis: prevalence and association with low back pain in the adult community-based population. Spine (Phila Pa 1976) 34:199-206, 2009

6. KwonBK, Berta S, Daffner SD, Vaccaro AR, Hilibrand AS, Grauer JN, et al: Radiographic analysis of transforaminal lumbar interbody fusion for the treatment of adult isthmic spondylolisthesis. J Spinal Disord Tech 16:469-476, 2003

7. Lee KH, Yue WM, Yeo W, Soeharno H, Tan SB: Clinical and radiological outcomes of open versus minimally invasive transforaminal lumbar interbody fusion. Eur Spine J 21:2265-2270, 2012

8. Lee SH, Choi WG, Lim SR, Kang HY, Shin SW: Minimally invasive anterior lumbar interbody fusion followed by percutaneous pedicle screw fi xation for isthmic spondylolisthesis. Spine J 4:644-649, 2004

9. Lee YG, Cha JH, Park JS: Clinical outcome of minimally invasive tubular retractor assisted microscopic discectomy in far lateral lumbar disc herniation. Korean J Spine 7:155-160, 2010

10. Liang B, Yin G, Zhao J, Li N, Hu Z: Surgical treatment of degenerative lumbar instability by minimally invasive transforaminal lumbar interbody fusion [abstract only]. Zhongguo Xiu Fu Chong Jian Wai Ke Za Zhi 25:1449-1454, 2011

11. McGirt MJ, Parker SL, Lerner J, Engelhart L, Knight T, Wang MY: Comparative analysis of perioperative surgical site infection after minimally invasive versus open posterior/transforaminal lumbar interbody fusion: analysis of hospital billing and discharge data from 5,170 patients. J Neurosurg Spine 14:771-778, 2011

12. Molinari RW, Sloboda JF, Arrington EC: Low-grade isthmic spondylolisthesis treated with instrumented posterior lumbar interbody fusion in US servicemen. J Spinal Disord Tech 18:S24-29, 2005
13. Pan J, Li L, Qian L, Zhou W, Tan J, Zou L, et al: Spontaneous slip reduction of low-grade isthmic spondylolisthesis following circumferential release via bilateral minimally invasive transforaminal lumbar interbody fusion: technical note and short-term outcome. Spine (Phila Pa 1976) 36:283-289, 2011

14. Recnik $G$, Košak R, Vengust R: Influencing segmental balance in isthmic spondylolisthesis using transforaminallumbar interbody fusion. J Spinal Disord Tech 26:246-251, 2013

15. Suh KT, Park WW, Kim SJ, Cho HM, Lee JS, Lee JS: Posterior lumbar interbody fusion for adult isthmic spondylolisthesis: a comparison of fusion with one or two cages. J Bone Joint Surg Br 90:1352-6, 2008

16. Tsahtsarlis A, Wood M: Minimally invasive transforaminal lumbar interbody fusion and spondylolisthesis. J Clin Neurosci 19: 858-861, 2012

17. Villavicencio AT, Burneikiene S, Nelson EL, Bulsara KR, Favors M, Thramann J: Safety of transforaminal lumbar interbody fusion and intervertebral recombinant human bone morphogenetic protein-2. J Neurosurg Spine 3:436-444, 2005

18. Wang J, Zhou Y, Feng Zhang Z, Qing Li C, Jie Zheng W, Liu J: Comparison of clinical outcome in overweight or obese patients after minimally invasive versus open transforaminal lumbar interbody fusion. J Spinal Disord Tech [Epub ahead of print]. 2012

19. Wang J, Zhou Y, Zhang ZF, Li CQ, Ren XJ, Chu TW, et al: Clinical study on lumbar spondylolisthesis treated by minimally invasive transforaminal lumbar interbody fusion [abstract only]. Zhonghua Wai Ke Za Zhi 49:1076-1080, 2011

20. Yan DL, Pei FX, Li J, Soo CL: Comparative study of PILF and TLIF treatment in adult degenerative spondylolisthesis. Eur Spine J 17:1311-1316, 2008 